\title{
On the Persistence and Predictability Properties of North Atlantic Climate Variability
}

\author{
CHRISTIAN FRANZKE \\ British Antarctic Survey, Cambridge, United Kingdom \\ TiM WOOLLINGS \\ Department of Meteorology, University of Reading, Reading, United Kingdom
}

(Manuscript received 22 March 2010, in final form 8 September 2010)

\begin{abstract}
The persistence and climate noise properties of North Atlantic climate variability are of importance for trend identification and assessing predictability on all time scales from several days to many decades. Here, the authors analyze these properties by applying empirical mode decomposition to a time series of the latitude of the North Atlantic eddy-driven jet stream. In previous studies, it has been argued that a slow decay of the autocorrelation function at large lags suggests potential extended-range predictability during the winter season. The authors show that the increased autocorrelation time scale does not necessarily lead to enhanced intraseasonal predictive skill. They estimate the fraction of interannual variability that likely arises due to climate noise as $43 \%-48 \%$ in winter and $70 \%-71 \%$ in summer. The analysis also indentifies a significant poleward trend of the jet stream that cannot be explained as arising from climate noise. These findings have important implications for the predictability of North Atlantic climate variability.
\end{abstract}

\section{Introduction}

The climate of the North Atlantic sector has a strong impact on European weather and climate, particularly through north-south shifts of the atmospheric eddydriven jet stream. The jet stream steers weather systems across the North Atlantic, determining the storm climate and influencing precipitation and temperature across large areas of Europe. In this study, we use a measure of the latitude of the jet stream as a proxy of North Atlantic climate variability to examine its climate noise, extendedrange predictability, and trend characteristics. Previous work in this area has focused on one aspect of jet stream variability, the North Atlantic Oscillation (NAO), and on whether its recent variations are significantly different from the characteristics of simple stochastic processes. For example, Wunsch (1999) showed that the winter NAO can be well represented as a first-order Markov process, although Stephenson et al. (2000) showed that a process with long-range dependence provides a better fit.

Corresponding author address: Dr. C. Franzke, British Antarctic Survey, High Cross, Madingley Road, Cambridge CB3 0ET, United Kingdom.

E-mail: chan1@bas.ac.uk
One simple measure of persistence and predictability is the autocorrelation function. In recent studies, it has been pointed out that the NAO exhibits unusual persistence, as evidenced by a "shoulder" of slow decay in the autocorrelation function between 10 and 30 days (e.g., Ambaum and Hoskins 2002; Rennert and Wallace 2009). However, Keeley et al. (2009) showed that this shoulder feature is sensitive to the presence of interannual variability, and as such it may not reflect enhanced predictability on intraseasonal time scales. The interannual variability itself is a combination of externally forced variations and variations arising from climate noise: that is, from sampling variability associated with the averaging of shorter time-scale intraannual fluctuations (Feldstein 2000, 2002; Franzke 2009; Keeley et al. 2009). The fraction of interannual variability, which is externally forced and hence potentially predictable, is of obvious interest for seasonal and longer-range forecasting. Here, we investigate these issues by applying an empirical mode decomposition (EMD) to an index of the jet stream latitude to separate variability on different time scales and examine their impact on extended-range predictability.

The relatively fast decorrelation of the NAO time series within the first 10 days has, along with the climate noise estimates, motivated a focus on synoptic time 
a)

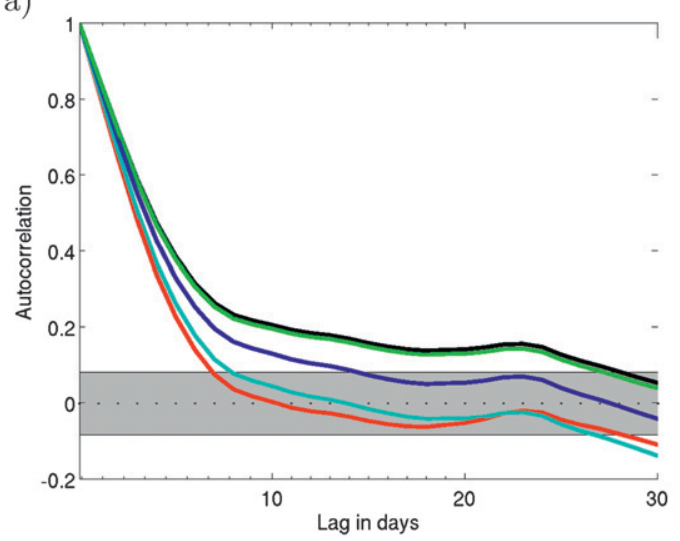

b)

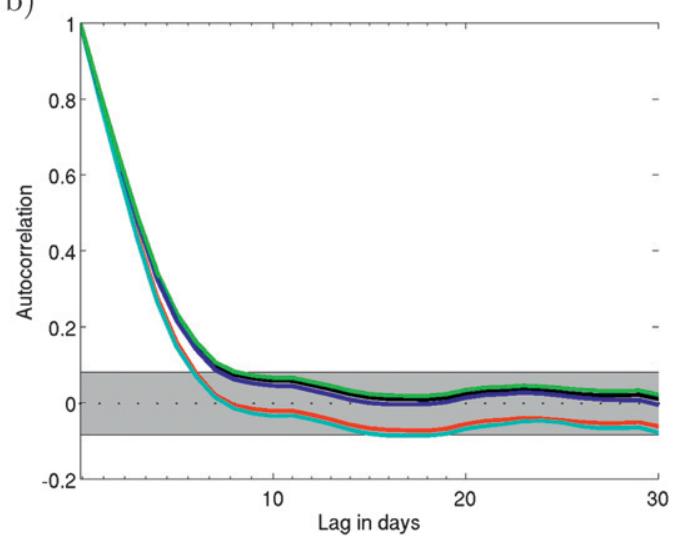

FIG. 1. Autocorrelation functions of daily JLI for (a) winter and (b) summer seasons. Black solid line: unfiltered JLI; green solid line: detrended (EMD trend) JLI; red solid line: intraseasonal (IMF 1-6) JLI; blue solid line: intraseasonal and interseasonal JLI (IMF 1-8); and cyan solid line: JLI with individual winter means subtracted. Autocorrelation values outside the shaded area are statistically significant at the $5 \%$ level.

scales in the study of the NAO (e.g., Benedict et al. 2004). However, this synoptic focus was challenged by Rennert and Wallace (2009), who suggested that the fast decorrelation results from the contamination of the daily NAO index by intermediate (6-30 day) frequency variability dominated by linear Rossby waves. This contamination was envisaged to arise from the partial projection of the wave patterns onto the spatial pattern of the NAO. The approach taken here of diagnosing the jet latitude may be less sensitive to the presence of wavelike variability than methods based on the projection of spatial patterns.

\section{North Atlantic climate variability index}

The jet latitude index (JLI) is a measure of the variability in the position of the eddy-driven jet stream over the North Atlantic region (Woollings et al. 2010). The JLI covers the period 1 December 1957 through 28 February 2002 using data from the 40-yr European Centre for Medium-Range Weather Forecasts (ECMWF) ReAnalysis (ERA-40; Uppala et al. 2005). The JLI is derived in the following way: 1) a mass-weighted average of the daily mean zonal wind is taken over the vertical levels $925,850,775$, and $700 \mathrm{hPa}$ and over the Atlantic sector $0^{\circ}-60^{\circ} \mathrm{W}$. 2) Winds poleward of $75^{\circ} \mathrm{N}$ and equatorward of $15^{\circ} \mathrm{N}$ are neglected. 3) The resulting wind field is low-pass filtered, only retaining periods greater than 10 days. 4) The JLI is defined as the latitude at which the maximum wind speed is found. 5) A smooth annual cycle is subtracted from the resulting time series. See Woollings et al. (2010) for more details, where it is also shown that this index describes jet stream variations that are associated with both the NAO and the east Atlantic (EA) teleconnection pattern and therefore represents a good general proxy of North Atlantic climate variability. In addition to its generality, the JLI has an advantage over patternbased indices such as the NAO in that it can be trivially calculated over all seasons with no complications arising from the changing of patterns during the annual cycle.

\section{Time scale and climate noise}

The autocorrelation function of the JLI is displayed as a black line in Figs. 1a,b for the winter and summer seasons, respectively. The autocorrelation function decays rather quickly in the first few days in both seasons. Although in summer the autocorrelation function decays to zero after about 10 days, in winter it stays at significantly nonzero values for up to lag 30 days. The plateau between lag 10 and lag 30 days of the related NAO autocorrelation function is usually referred to as the shoulder feature, and it was attributed to interannual variability by Keeley et al. (2009). Here, we examine the contributions of different time scales to this autocorrelation function plateau and assess if it potentially enhances predictability. For this purpose, we utilize advanced timeseries methods.

The EMD is a recently developed algorithm (Huang et al. 1998; Huang and Wu 2008; Franzke 2009, 2010) to decompose a univariate time series into a finite number of components called intrinsic mode functions (IMFs),

$$
x(t)=\sum_{j=1}^{M} \psi_{j}(t)+R(t),
$$

where the $j$ th IMF $\psi_{j}$ can be written in polar coordinates $\psi_{j}(t)=r_{j}(t) \sin \left[\theta_{j}(t)\right]$ where $r_{j}$ is the $j$ th amplitude, $\theta_{j}$ is 
the $j$ th instantaneous frequency, and $R$ the instantaneous mean. Both the amplitude and frequency are time dependent and thus different from Fourier modes where both $r_{j}$ and $\theta_{j}$ are time independent. An IMF is defined by the following two properties (Huang et al. 1998): 1) each IMF $\psi_{j}$ has exactly one zero crossing between two consecutive local extrema and 2) the mean of each IMF $\psi_{j}$ is zero. A detailed description of the algorithm is given by Huang et al. (1998) and Franzke (2009). Here, we use the same setup as in Franzke (2009, 2010). The major advantage of EMD over standard wavelet and Fourier analyses is that it is a data adaptive methodology, whereas wavelet and Fourier analyses use a priori defined basis functions. See Huang et al. (1998) for an extensive discussion of the advantages of EMD over wavelet and Fourier analysis. The ability and robustness of EMD to extract nonlinear trends from noisy time series has been demonstrated by Franzke (2010), though it should be remembered that any definition of a trend depends on the method used to identify it.

The JLI has been decomposed into IMFs and an instantaneous mean, which we will interpret as a trend in this study and will be called the EMD trend. Each IMF mode has a mean period that is defined here as the average time between two local maxima. This analysis reveals that IMF modes 1-6 correspond to intraseasonal variability and IMFs 9-12 correspond to interannual variability (Fig. 2 and Table 1). This allows us to use EMD as a nonlinear filter and utilize the IMFs to define the following frequency bands: intraseasonal with mean periods less than 90 days (sum of IMFs 1-6), interseasonal with mean periods between 90 and 365 days (IMFs 7-8), and interannual with mean periods larger than 365 days (IMFs 9-12). The different filtered time series are uncorrelated and thus orthogonal. The intraseasonal, interseasonal, and interannual bands explain $89.5 \%, 7.7 \%$, and $2.8 \%$ of the total variability, respectively.

The EMD analysis also reveals a statistically significant trend that is different from a linear least squares fit (Fig. 2n). The EMD trend is steeper than the linear least squares trend in the period 1957-87 and then levels off after this. The trend is associated with a poleward shift of the jet stream, which is consistent in sign with the projected jet stream changes in Intergovernmental Panel on Climate Change (IPCC) scenario climate model projections (Yin 2005; Lorenz and DeWeaver 2007).

Most studies assume that climate variability can be reasonably well represented by a first-order Markov process in the form of a simple autoregressive process of first-order [AR(1)] (e.g., Wilks 1995; Wunsch 1999; Feldstein 2000; Masato et al. 2009). This process has a memory depth of 1 ; that is, to predict the next value one only needs to know the current value, and thus knowledge of past values will not increase the predictive skill. However, this can be a problematic choice, as recently shown by Keeley et al. (2009), because it can overestimate the autocorrelation time scale. A more flexible approach is to use an autoregressive moving average (ARMA) model (Jones 1980; Wilks 1995), which is given by

$$
\left(1-\sum_{i=1}^{p} \phi_{i} L^{i}\right) x_{t}=\left(1+\sum_{i=1}^{q} \theta_{i} L^{i}\right) \zeta_{t}
$$

where $x_{t}$ indicates the state variable at time $t ; L$ denotes the lag operator $L^{k} x_{t}=x_{t-k}$; and $p$ and $q$ are the orders of the autoregressive and moving average parts, respectively. Here, $\zeta_{t}$ is standard Gaussian distributed white noise and $\phi$ and $\theta$ are the autoregressive and moving average coefficients. By setting $p=1$ and $q=0$, the $\operatorname{ARMA}(p, q)$ model simplifies to the standard $\operatorname{AR}(1)$ model. We utilize the ARMA process to get a rough estimate of the memory depth of the JLI. We denote as memory depth the order of the $\operatorname{ARMA}(p, q)$ model; that is, the larger of $p$ and $q$ denotes the memory depth. The optimal model order is estimated using the algorithm of Broersen (2000). The unfiltered time series has $p=3$ and $q=2$ for the winter [DecemberFebruary (DJF)], spring [March-May (MAM)] and summer [June-August (JJA)] seasons and $p=2$ and $q=1$ for autumn [September-November (SON)]; thus, the memory depth is 3 for both the winter and summer seasons. The orders reduce to $p=2$ and $q=1$ for the intraannual filtered time series so that the memory depth is 2 . This suggests that daily climate indices are not necessarily firstorder Markov processes.

We now test the statistical significance of the individual IMF modes by using the seasonally varying ARMA model to model the intraseasonal JLI variability (Franzke 2009). Here, we assume the null hypothesis that all IMFs are indistinguishable from the seasonal ARMA model. The coefficients of the ARMA model have been derived from the intraseasonally filtered data because the aim is to determine whether the variations on interannual time scales are distinct from sampling variability of the intraannual variations: that is, distinct from climate noise (Franzke 2009). Because the fraction of variance due to climate noise is unknown, we performed two different sets of ARMA simulations. The first set (ARMA1) has the same variance as the sum of the IMF modes of the JLI with intraseasonal periods (IMFs 1-6), whereas in the second set, denoted as ARMA2, the variance of ARMA1 has been rescaled to fit the variance of the JLI. In ARMA2, we assume that all JLI variability is caused 
a) Jet Latitude Index

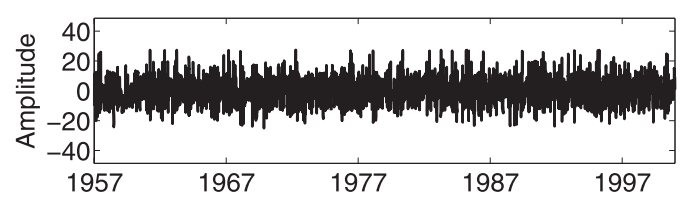

b) IMF-1 (11.1\%; 4 days)

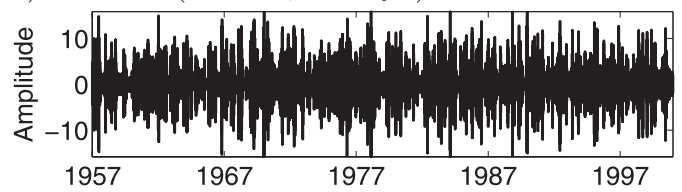

c) IMF-2 (19.8\%; 8 days $)$

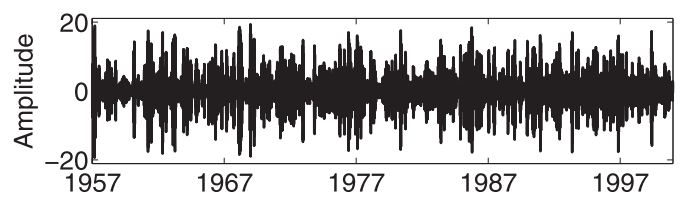

d) IMF-3 (19.4\%; 15 days)

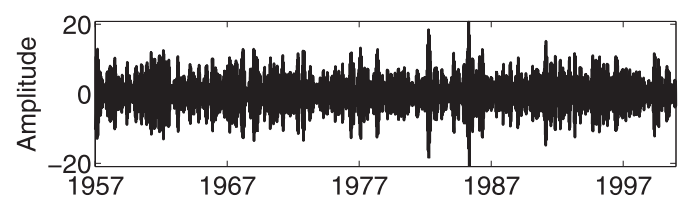

e) IMF-4 (17.3\%; 25 days $)$

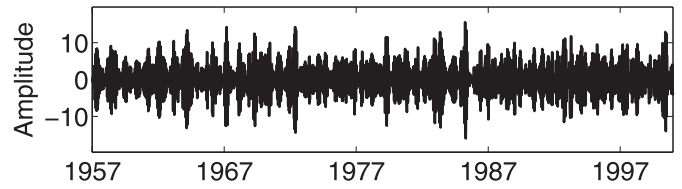

f) $\operatorname{IMF}-5(13.0 \% ; 45$ days $)$

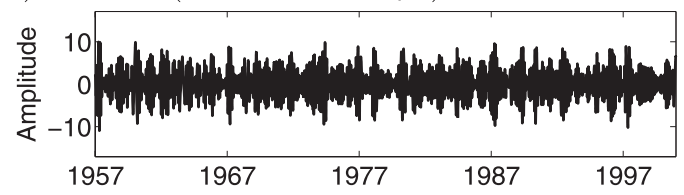

g) IMF-6 (8.5\%; 81 days $)$

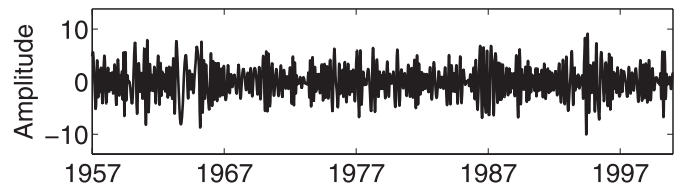

h) IMF-7 (4.8\%; 146 days)

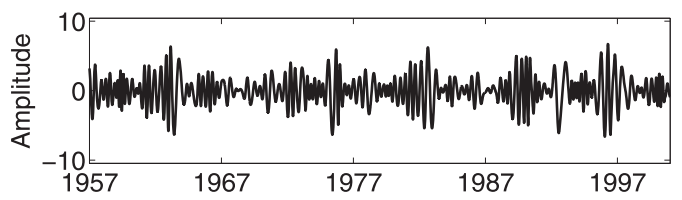

i) IMF-8 $(2.6 \% ; 277$ days $)$

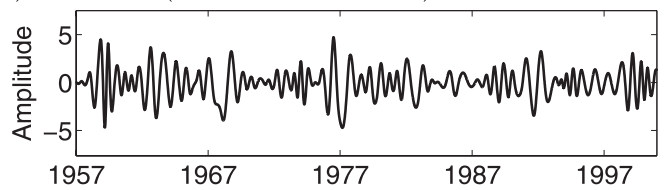

j) IMF-9 (1.8\%; 553 days)

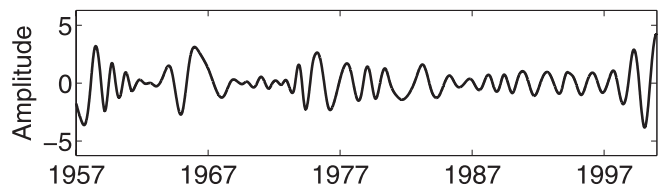

k) IMF-10 (1.1\%; 1373 days $)$

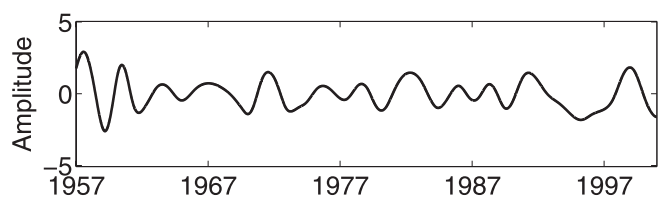

1) $\operatorname{IMF}-11(0.4 \% ; 3212$ days $)$

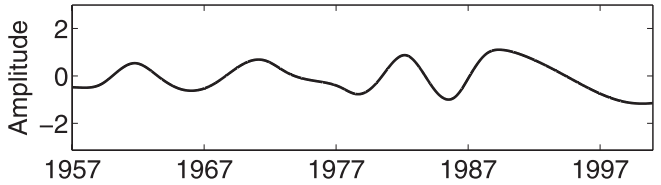

m) IMF-12 (0.03\%; 8029 days)

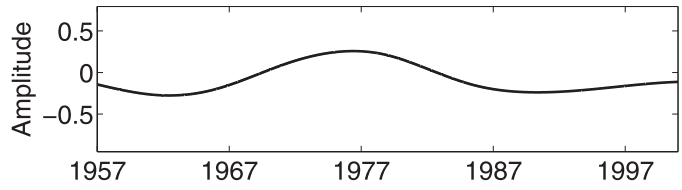

n) Residual

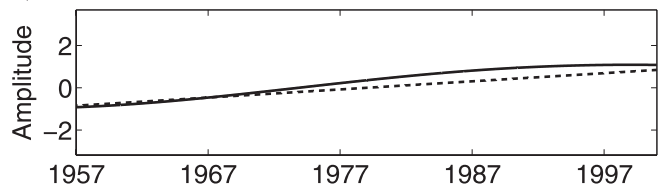

FIG. 2. IMFs of EMD for the daily JLI. (n) The dashed line is the linear least squares fit of the JLI. The numbers in parentheses are the explained variance and the mean period of the IMFs, respectively.

by short-term (intraseasonal) fluctuations. This test uses a Mahalanobis metric (Mahalanobis 1936), which takes into account both the mean period and the variance of the respective IMFs. The results are displayed in Table 1 and reveal that IMF modes 1-3, 5-7, and 9-10 and the instantaneous mean are significantly different from the best-fit seasonally varying ARMA process at the $2.5 \%$ level for ARMA1, whereas for ARMA2 only the first three IMFs and the instantaneous mean are significantly different at the $2.5 \%$ level. Although the intraseasonal IMFs explain almost $90 \%$ of the JLI variance, rescaling the ARMA1 model to have the JLI variance (ARMA2) negates the statistical significance of the interannual IMFs. This suggests that climate noise is potentially able to explain most of the interannual variability. Furthermore, the IMF modes that are significant against both 
TABLE 1. Mean periods of JLI and corresponding seasonal ARMA $(p, q)$ process in days. The mean period is defined as the mean distance between two consecutive maxima. IMF modes significant at the $2.5 \%$ level are highlighted by an X in rows 3 and 4 . For rows 3 and 4, ARMA processes with variance equal to the corresponding intraseasonal (for ARMA1) and total (for ARMA2) variance have been used.

\begin{tabular}{lccccccccccrrr}
\hline \hline \multicolumn{1}{c}{ IMF } & 1 & 2 & 3 & 4 & 5 & 6 & 7 & 8 & 9 & 10 & 11 & 12 & Residual \\
\hline Mean period JLI & 4 & 8 & 15 & 25 & 45 & 81 & 146 & 277 & 553 & 1373 & 3212 & 8029 & 16059 \\
Mean period ARMA & 3 & 6 & 12 & 24 & 45 & 86 & 170 & 337 & 675 & 1367 & 2772 & 5868 & 9078 \\
Significant ARMA1 & X & X & X & & X & X & X & & X & X & X \\
Significant ARMA2 & X & X & X & & & & & & & & X \\
\hline
\end{tabular}

ARMA models can be considered to be robust. The fact that the EMD trend is statistically significant when tested against both ARMA1 and ARMA2 suggests that the EMD trend is very robust and cannot be explained as arising from climate noise. Thus, the EMD trend is likely to have arisen because of external forcing, such as oceanic forcing, cryospheric forcing, or anthropogenic warming.

The existence of a statistically significant trend raises the question of whether the shoulder feature in the autocorrelation function could be due to the trend. However, the autocorrelation function of the detrended JLI (Fig. 1a, green line) shows a very similar behavior to the autocorrelation function of the raw JLI; therefore, the shoulder feature is not an artefact of the trend.

Now we assess whether certain frequency bands are responsible for the shoulder feature. The autocorrelation function of the sum of IMF 1-6 (intraseasonal modes) shows a rapid decay to insignificant values at about lag 7 days (Fig. 1a, red line). The autocorrelation function of the sum of IMF 1-8 (intraannual modes; Fig. 1a, blue line) also has a weaker shoulder than the unfiltered JLI, showing that all three frequency band contributions are needed to explain the slow decay of the unfiltered JLI. This suggests that although the interseasonal and interannual variability only explain about $10 \%$ of the total variance, they have a large impact on the decay of the autocorrelation function at long lags. This is in good agreement with the results of Keeley et al. (2009), who subtracted individual seasonal means from the data before calculating the autocorrelation function. For reference, we applied the same procedure, and the result (Figs. 1a,b; cyan line) is very close to the intraseasonal autocorrelation function for both summer and winter and is even closer to the combination of intraseasonal and interannual autocorrelation function for the winter season (not shown). The results are very similar for the summer season, with the exception that the interannual frequency band makes very little contribution to the autocorrelation function of the unfiltered JLI.

Next we utilize seasonal ARMA models fitted to the JLI (IMF 1-12; ARMA3) and to IMF 1-8 (intraannual modes; ARMA4) to generate surrogate time series and calculate autocorrelation functions to compare their decay with the JLI. Figures 3a,b show that these ARMA models provide an excellent fit to the autocorrelation functions apart from for the unfiltered case in winter at long lags. In this case, both ARMA models capture the initial decay of the respective indices well until lag 9 days but not afterward. We calculate significance levels for the autocorrelation functions by using ensembles of ARMA realizations with 1000 members for each of the 44 seasons. This allows us to empirically estimate the 2.5 and 97.5 percentiles from the ensemble. The ARMA models can produce significant autocorrelation values up to lag 20 days, which is less than the observed unfiltered JLI but also much longer than the mean autocorrelation function of the ARMA ensembles, which is computed as the average over all autocorrelation functions of the ensemble. This sampling uncertainty therefore suggests that part of the shoulder feature could in fact stem from intraseasonal fluctuations.

We also calculated rough estimates for the fraction of interannual variability, which likely arises because of climate noise. The climate noise fraction is estimated from an ensemble of 1000 members of the seasonal ARMA1 and ARMA2 models as the ratio between the respective ARMA interannual variability, which is calculated from seasonal averages of the daily surrogate data, and the variance of seasonal means of the JLI. By doing so, we estimate that during winter about $43 \%-48 \%$ and during summer about $70 \%-71 \%$ of interannual variability is due to climate noise. In both seasons the lower value corresponds to the ARMA1 and the higher value to the ARMA2 estimate.

\section{Predictability}

The slow decay of the autocorrelation function of the unfiltered JLI suggests enhanced extended-range predictability. Here, we test whether this is indeed the case. For this purpose, we perform predictability experiments by utilizing the ARMA1 model. We carry out leave-oneout hindcast experiments; that is, for predicting the winter of 2000 we use all winter seasons but 2000 to fit the ARMA1 parameters and then perform predictability 
a)

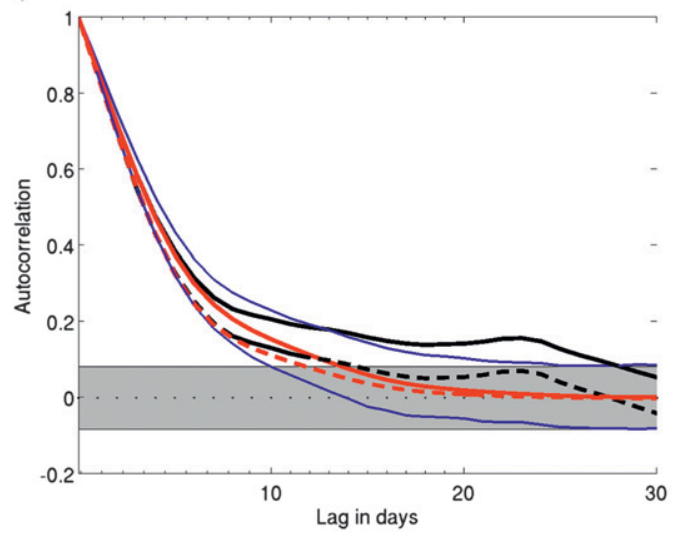

b)

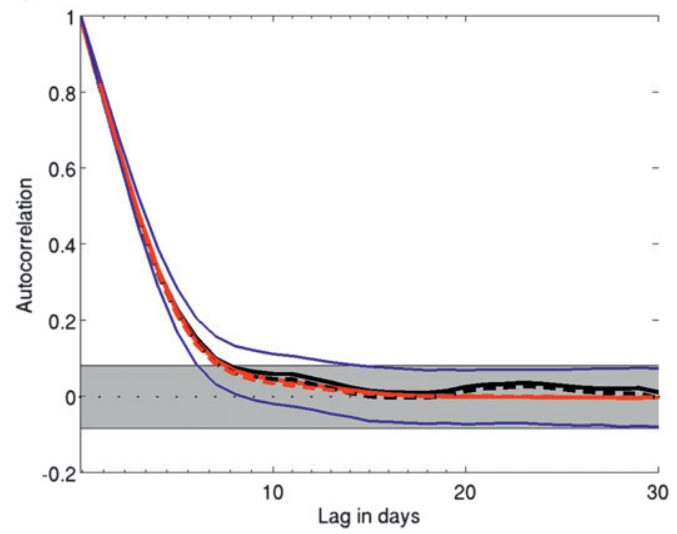

FIG. 3. Autocorrelation functions for (a) winter and (b) summer season. Black solid line: unfiltered JLI; red solid line: ensemble-mean ARMA3 of unfiltered JLI; black dashed line: intraannual JLI; and red dashed line: ensemble mean ARMA4 of intraannual JLI. The thin blue lines indicate the $2.5 \%$ and $97.5 \%$ percentiles of ensembles of 1000 ARMA3 simulations fitted to the JLI (the percentiles for the ARMA4 ensembles are very similar). Autocorrelation values outside the shaded area are statistically significant at the $5 \%$ level.

experiments for the winter of 2000. We now perform two sets of hindcast experiments: 1) where we hindcast the intraannual JLI using the ARMA1 model and 2) where we assume perfect predictability of the interannual variability (e.g., IMF 9-12) but use the same ARMA1 model as for set 1 . To compare both sets of hindcast experiments we use the skill score $\mathrm{SS}=1-\left(\mathrm{MSE}_{1} / \mathrm{MSE}_{2}\right)$ (Wilks 1995), where $\mathrm{MSE}_{i}$ denotes the mean square error between hindcast experiment $i$ and the unfiltered JLI averaged over all winter seasons and all starting days of the hindcasts. The skill score is calculated for predictions of particular days but also for 10-day averages out to a lead time of 30 days. We do ensemble hindcasts with the ARMA model with 100 ensemble members with different noise realizations and then use the ensemble mean for the hindcast skill. Both sets of hindcast experiments have about the same mean square error; thus, the skill score is close to zero (not shown). Using both interseasonal and intraannual IMFs (IMF 7-12) for set 2 of the hindcast experiments gives very similar results. This suggests that the shoulder feature of the autocorrelation function does not imply enhanced predictability on intraseasonal time scales when the underlying process generating the intraseasonal variability is represented by an ARMA model. Using the ARMA4 model gives qualitatively very similar results.

\section{Concluding discussion}

Our main results are as follows:

- The "shoulder" feature in the autocorrelation function does not lead to enhanced intraseasonal predictability in hindcast experiments with a seasonal ARMA model, even if the time scales that lead to the shoulder are assumed to be perfectly predictable.

- The JLI exhibits a significant poleward trend over the ERA-40 period that does not arise from climate noise. - About $97 \%$ of JLI variability is in the intraannual range. - In winter and summer, $43 \%-48 \%$ and $70 \%-71 \%$ of JLI interannual variability, respectively, is likely due to climate noise.

These results have important implications for the predictability of North Atlantic weather and climate on several time scales. With respect to prediction on seasonal and longer time scales, we estimate that the potentially predictable, externally forced variability comprises about $52 \%-57 \%$ of the total interannual variance in winter and $29 \%-30 \%$ in summer. In winter, this estimate is slightly lower than that of about $70 \%$ suggested by Keeley et al. (2009). In summer, however, our estimate is higher, which may reflect the influence of the Atlantic multidecadal oscillation on the summer NAO on interdecadal time scales (Folland et al. 2009).

Although a large fraction of interannual variability is potentially predictable, its effective predictability appears to be low because much of the jet stream variability occurs on intraannual time scales. With respect to extended-range weather forecasting, the jet stream location is not predictable on horizons longer than a week. Note, however, that the autocorrelation function provides an average measure of predictability, which may be higher at some times (e.g., during blocking; Masato et al. 2009) and of course lower at other times. 
Acknowledgments. This study is part of the British Antarctic Survey Polar Science for Planet Earth Programme. It was funded by The Natural Environment Research Council. We thank the European Centre for Medium-Range Weather Forecasts for providing the ERA-40 and the anonymous reviewers for their constructive comments, which helped to improve the manuscript.

\section{REFERENCES}

Ambaum, M. H. P., and B. J. Hoskins, 2002: The NAO tropospherestratosphere connection. J. Climate, 15, 1969-1978.

Benedict, J. J., S. Lee, and S. B. Feldstein, 2004: Synoptic view of the North Atlantic Oscillation. J. Atmos. Sci., 61, 121-144.

Broersen, P. M. T., 2000: Autoregressive model orders for Durbin's MA and ARMA estimators. IEEE Trans. Acoust., Speech, Signal Process., 48, 2454-2457.

Feldstein, S. B., 2000: The timescale, power spectra, and climate noise properties of teleconnection patterns. J. Climate, 13, 4430-4440.

_ 2002: The recent trend and variance increase of the annular mode. J. Climate, 15, 88-94.

Folland, C. K., J. Knight, H. W. Linderholm, D. Fereday, S. Ineson, and J. W. Hurrell, 2009: The summer North Atlantic Oscillation: Past, present, and future. J. Climate, 22, 1082-1103.

Franzke, C., 2009: Multi-scale analysis of teleconnection indices: Climate noise and nonlinear trends. Nonlinear Processes Geophys., 16, 65-76.

- 2010: Long-range dependence and climate noise characteristics of Antarctic temperature data. J. Climate, 23, 6073 6080.

Huang, N. E., and Z. Wu, 2008: A review on Hilbert-Huang transform: Method and its applications to geophysical studies. Rev. Geophys., 46, RG2006, doi:10.1029/2007RG000228. and Coauthors, 1998: The empirical mode decomposition and the Hilbert spectrum for nonlinear and non-stationary time series analysis. Proc. Roy. Soc. London, 454A, 903-995.

Jones, R. H., 1980: Maximum likelihood fitting of ARMA models of time series with missing observations. Technometrics, 22, 389-395.

Keeley, S. P. E., R. T. Sutton, and L. C. Shaffrey, 2009: Does the North Atlantic Oscillation show unusual persistence on intraseasonal timescales? Geophys. Res. Lett., 36, L22706, doi:10.1029/2009GL040367.

Lorenz, D. J., and E. T. DeWeaver, 2007: Tropopause height and zonal wind response to global warming in the IPCC scenario integrations. J. Geophys. Res., 112, D10119, doi:10.1029/ 2006JD008087.

Mahalanobis, P. C., 1936: On the generalised distance in statistics. Proc. Natl. Inst. Sci. India, 2, 49-55.

Masato, G., B. Hoskins, and T. Woollings, 2009: Can the frequency of blocking be described by a red noise process? J. Atmos. Sci., 66, 2143-2149.

Rennert, K. J., and J. M. Wallace, 2009: Cross-frequency coupling, skewness, and blocking in the Northern Hemisphere winter circulation. J. Climate, 22, 5650-5666.

Stephenson, D. B., V. Pavan, and R. Bojariu, 2000: Is the North Atlantic Oscillation a random walk? Int. J. Climatol., 20,1-18.

Uppala, S. M., and Coauthors, 2005: The ERA-40 Re-Analysis. Quart. J. Roy. Meteor. Soc., 131, 2961-3012.

Wilks, D. S., 1995: Statistical Methods in the Atmospheric Sciences. Academic Press, 464 pp.

Woollings, T., A. Hannachi, and B. Hoskins, 2010: Variability of the North Atlantic eddy-driven jet stream. Quart. J. Roy. Meteor. Soc., 136, 856-868.

Wunsch, C., 1999: The interpretation of short climate records, with comments on the North Atlantic and Southern Oscillations. Bull. Amer. Meteor. Soc., 80, 245-255.

Yin, J. H., 2005: A consistent poleward shift of the storm tracks in simulations of 21st century climate. Geophys. Res. Lett., 32 , L18701, doi:10.1029/2005GL023684. 